Article

\title{
Associations between Dietary Iron and Zinc Intakes, and between Biochemical Iron and Zinc Status in Women
}

\section{Karen Lim ${ }^{1}$, Alison Booth ${ }^{1}$, Ewa A. Szymlek-Gay ${ }^{1}$, Rosalind S. Gibson ${ }^{2}$, Karl B. Bailey ${ }^{2}$, David Irving ${ }^{3,4}$, Caryl Nowson ${ }^{1}$ and Lynn Riddell ${ }^{1, *}$}

1 Centre for Physical Activity and Nutrition Research, School of Exercise and Nutrition, Deakin University, 221 Burwood Highway, Burwood, Victoria 3125, Australia;

E-Mails: k.lim@deakin.edu.au (K.L.); alison.booth@deakin.edu.au (A.B.);

ewa.szymlekgay@deakin.edu.au (E.A.S.-G.); caryl.nowson@deakin.edu.au (C.N.)

2 Department of Human Nutrition, University of Otago, PO Box 56, Dunedin 9054, New Zealand;

E-Mails: rosalind.gibson@otago.ac.nz (R.S.G.); karl.bailey@otago.ac.nz (K.B.B.)

3 Australian Red Cross Blood Service, 17 O’Riordan Street, Alexandria, New South Wales 2015, Australia; E-Mail: dirving@redcrossblood.org.au

4 University of Technology, Sydney 15 Broadway, Ultimo, New South Wales 2007, Australia

* Author to whom correspondence should be addressed; E-Mail: lynn.riddell@deakin.edu.au; Tel.: +61-3-9251-7270; Fax: +61-3-9244-6017.

Received: 25 March 2015 / Accepted: 14 April 2015 / Published: 20 April 2015

\begin{abstract}
Iron and zinc are found in similar foods and absorption of both may be affected by food compounds, thus biochemical iron and zinc status may be related. This cross-sectional study aimed to: (1) describe dietary intakes and biochemical status of iron and zinc; (2) investigate associations between dietary iron and zinc intakes; and (3) investigate associations between biochemical iron and zinc status in a sample of premenopausal women aged 18-50 years who were recruited in Melbourne and Sydney, Australia. Usual dietary intakes were assessed using a 154-item food frequency questionnaire $(n=379)$. Iron status was assessed using serum ferritin and hemoglobin, zinc status using serum zinc (standardized to 08:00 collection), and presence of infection/inflammation using C-reactive protein $(n=326)$. Associations were explored using multiple regression and logistic regression. Mean (SD) iron and zinc intakes were 10.5 (3.5) mg/day and 9.3 (3.8) mg/day, respectively. Median (interquartile range) serum ferritin was $22(12-38) \mu \mathrm{g} / \mathrm{L}$ and mean serum zinc concentrations (SD) were $12.6(1.7) \mu \mathrm{mol} / \mathrm{L}$ in fasting samples and $11.8(2.0) \mu \mathrm{mol} / \mathrm{L}$ in nonfasting samples. For each $1 \mathrm{mg} /$ day increase in dietary iron intake, zinc intake increased by $0.4 \mathrm{mg} / \mathrm{day}$. Each $1 \mu \mathrm{mol} / \mathrm{L}$ increase in serum zinc corresponded to a
\end{abstract}


$6 \%$ increase in serum ferritin, however women with low serum zinc concentration (AM fasting $<10.7 \mu \mathrm{mol} / \mathrm{L}$; AM nonfasting $<10.1 \mu \mathrm{mol} / \mathrm{L}$ ) were not at increased risk of depleted iron stores (serum ferritin $<15 \mu \mathrm{g} / \mathrm{L} ; p=0.340$ ). Positive associations were observed between dietary iron and zinc intakes, and between iron and zinc status, however interpreting serum ferritin concentrations was not a useful proxy for estimating the likelihood of low serum zinc concentrations and women with depleted iron stores were not at increased risk of impaired zinc status in this cohort.

Keywords: iron; zinc; women; minerals; nutritional status

\section{Introduction}

The 2011-2012 Australian Health Survey indicated that 12\% of women 16-44 years of age had depleted iron stores (serum ferritin < $15 \mu \mathrm{g} / \mathrm{L}$ [1]) compared to 1\%-2\% of Australian men [2]. Premenopausal women with iron stores at this level are more likely to experience impaired physical performance [3], and there is some evidence that cognitive ability may also be negatively affected [4,5]. As iron is found in similar food sources to zinc [6,7], inadequate dietary iron and zinc intakes may occur simultaneously [8]. There is also evidence that the form of iron and zinc in plant-based foods is less readily available for absorption than from diets containing meat, perhaps more so than other trace minerals [9,10]. It is widely acknowledged that the inorganic form of iron, non-heme iron, is less bioavailable than heme iron [11], and it has been hypothesized that analogous to non-heme iron, inorganic zinc salts in plants are a less bioavailable form of dietary zinc [12,13]. Moreover, compounds such as phytate may further impede absorption of both iron and zinc from foods $[9,10]$. Due to these diet-based similarities, there is potential for iron and zinc status to be positively correlated [14-17], however there are no data on zinc status to explore this relationship in Australian women. Assessment of zinc status is notoriously difficult [18], however as zinc plays a crucial role in immune function [19] and may be linked to depression [20], assessing zinc status to ensure optimal nutrition is important to health. Serum zinc, the most commonly used biomarker of zinc status, undergoes diurnal variation and is reduced following a meal [21]. As circulating zinc is present in such low concentrations, contamination of serum samples is a concern, and trace element-free equipment is required [21]. Given the challenges associated with measuring zinc status, understanding the relationship between biochemical measures of iron and zinc may provide an opportunity to use iron status as a proxy to screen women to assess their risk of impaired zinc status. Three studies in premenopausal women conducted in industrialized countries have reported correlations between serum ferritin and serum zinc concentrations ranging from 0.10 to $0.45[14,16,17]$, and one has found significantly lower serum ferritin concentration in women with low zinc status (mean serum ferritin 10.8 (SD 7.2) $\mu \mathrm{g} / \mathrm{L}$ vs. 26.0 (SD 15.3) $\mu \mathrm{g} / \mathrm{L}$ ) [15]. These findings indicate there may be an association between iron and zinc status, however only one study [17] accounted for infection and inflammation, which are known to elevate serum ferritin and suppress serum zinc concentrations [1,21]. Investigating the association between iron and zinc status whilst accounting for inflammation may further our understanding of the relationship between these two nutrients and 
provide some insight into whether depleted iron stores may predict risk of impaired zinc status among Australian women.

Our aims for this study were to: (1) describe dietary intakes and biochemical status of iron and zinc; (2) investigate associations between dietary iron and zinc intakes; and (3) investigate associations between biochemical iron and zinc status in a sample of premenopausal women aged 18-50 years in Australia.

\section{Methods}

\subsection{Study Design}

Women 18-50 years of age were recruited for this cross-sectional study in three phases: (a) from the student and staff population at Deakin University (Burwood, Melbourne, Australia) between July and November 2010 ( $n=51$ ); (b) from blood donors registered with the Australian Red Cross Blood Service (Blood Service; Sydney, Australia) between November 2010 and May 2011 ( $n=203$ ); and (c) from the student and staff population at Deakin University (Burwood, Melbourne, Australia) and residents of metropolitan Melbourne between July 2012 and January $2014(n=142)$. Written informed consent was obtained from all participants and ethical approval was granted by the Deakin University Human Research Ethics Committee (references 2009-191 and 2012-046) and the Blood Service Ethics Committee (reference 2010\#01).

\subsection{Recruitment and Eligibility}

Recruitment at the Deakin University Burwood campus was conducted via flyers, announcements in lectures, and on the university's online learning portal. Recruitment at the Blood Service was conducted as part of routine phone calls reminding registered blood donors when they are eligible to make their next blood donation. Recruitment of Melbourne residents was conducted via Facebook ('sharing' of a Facebook page about the study and paid advertisements of this page within Facebook) and printed advertisements in local newspapers.

Across all recruitment phases, women who had been through menopause, who were currently pregnant or lactating, or had been in the past 6 months, were ineligible to participate. In the recruitment period from 2012 to 2014, we introduced a criterion to exclude women who had donated blood in the previous 12 months as a number of blood donors had been recruited from 2010 to 2011.

\subsection{Measures}

For Melbourne-based recruitment, data was collected at the Deakin University Burwood campus. For Blood Service recruitment, data was collected at the Blood Service collection center when the women presented to make their scheduled blood donation. Data collection involved paper-based questionnaires and a blood sample. 


\subsubsection{Anthropometric, Demographic, and Blood Donation Characteristics}

Participants recruited in Melbourne had height and weight directly measured. Height was measured to $0.1 \mathrm{~cm}$ using a wall-mounted stadiometer and weight measured to $0.1 \mathrm{~kg}$ using electronic scales. Women recruited at the Blood Service self-reported height via questionnaire and had weight measured to $1 \mathrm{~kg}$ as per the Blood Service standard practice. Body mass index (BMI) was calculated as $\mathrm{kg} / \mathrm{m}^{2}$ and interpreted using World Health Organization cut-offs for adults, with normal range being 18.5 to $24.9 \mathrm{~kg} / \mathrm{m}^{2}$, overweight being 25.0 to $29.9 \mathrm{~kg} / \mathrm{m}^{2}$, and obese being $>30 \mathrm{~kg} / \mathrm{m}^{2}$ [22]. All participants completed a paper-based questionnaire to report their age, education level, cigarette smoking status, and use of oral contraception (protective against impaired iron status [23] and associated with suppressed serum zinc concentration [21]).

Women recruited in Melbourne in 2010 self-reported blood donor status and frequency of donation via questionnaire. Blood donation history of women recruited at the Blood Service was sourced from Blood Service records. From this information, women were dichotomized as having/not having made a donation in the previous 12 months. The amount of blood donated was quantified using conversion factors. Each whole blood donation was considered to be $470 \mathrm{~mL}$ and each apheresis (plasmapheresis and plateletpheresis) donation was considered to be $60 \mathrm{~mL}$ of whole blood. Blood donation was also considered as a continuous variable of $\mathrm{mL}$ donated in the past year.

\subsubsection{Dietary Intake}

Participants' usual dietary intakes were recorded using the Cancer Council Victoria Dietary Questionnaire for Epidemiological Studies v3.1 (DQES v3.1), a paper-based semiquantitative 154-item food frequency questionnaire (FFQ) that assesses the consumption frequency of 140 foods and beverages over the previous 12 months. The previous version of this FFQ [24] has been validated against seven-day weighed food records, with Bland Altman analyses finding mean difference of $-0.22 \mathrm{mg} / \mathrm{day}$ and $95 \%$ CI limits of agreement of -8.1 to 7.7 for dietary iron intake, and mean difference of -0.82 and 95\% CI limits of agreement of -7.2 to 5.6 for dietary zinc intake [25]. Pearson correlation coefficients of intakes from the food records and DQES v2 was $r=0.44$ for dietary iron and $r=0.40$ for dietary zinc [25].

Women reported their consumption frequency over the past 12 months by choosing from 10 frequency categories ranging from 'never' to 'three or more times per day'. Portion sizes were calibrated using photographs of scaled portions of six foods, and two questions regarding overall daily consumption of fruits and vegetables were used to calibrate consumption of individual fruits and vegetables. The nutritional analysis of the FFQ was conducted independently of the Cancer Council Victoria. The consumption frequency categories were converted to numerical daily equivalent frequencies (e.g., never $=0$, once per week $=0.14$, once per day $=1$, three or more times per day $=3$ ), which were used in conjunction with portion information to estimate the usual amount of food or beverage item consumed as g/day. Usual nutrient intake was then calculated using Australian nutrient databases NUTTAB 2010 [26] and AUSNUT 2007 [27] via FoodWorks 7 (Xyris Software, Queensland, Australia). Dietary data was available for 382 women. Three women who completed the FFQ reported energy intakes $>3$ SD above 
the mean, and were excluded from descriptive and inferential dietary analyses $[28,29]$. No women reported energy intakes $<3 \mathrm{SD}$ below the mean.

\subsubsection{Supplement Intake}

Intake of dietary supplements was self-reported. Participants were asked to record the name of any dietary supplements they were currently using, the frequency of consumption, and the dose. Supplements were characterized as multivitamin/mineral supplements containing iron, multivitamin/mineral supplements containing zinc, iron-specific supplements, or zinc-specific supplements, and the dose of elemental iron or zinc was confirmed by checking supplement packaging or the manufacturers' websites. To estimate supplemental iron and zinc intake as mg/day, the total $\mathrm{mg}$ of iron and zinc from all supplements consumed per week was divided by seven days. Use of supplements containing iron or zinc was also investigated as a dichotomous variable.

\subsubsection{Assessing Adequacy of Dietary Intakes}

Due to the wide inter-individual variation in menstrual losses, iron requirements in premenopausal women are distributed asymmetrically around the median, thus unlike most nutrients, the cutpoint method of assessing intakes against the Estimated Average Requirement (EAR) for iron (i.e., the amount estimated to meet the requirements of half the healthy individuals in the group) cannot be used to estimate the prevalence of inadequate intakes [30]. The adequacy of dietary iron intakes of the cohort was therefore evaluated using the probability approach [30]. This approach quantifies the prevalence of inadequate iron intakes in a cohort by comparing the cohort's distribution of usual iron intakes to a proposed distribution of iron requirements [30]. The Institute of Medicine (IOM) [23] has published 14 levels of usual iron intake associated with probabilities of inadequacy for cohorts of women based on oral contraceptive use (cohort not using oral contraception, cohort using oral contraception, and mixed cohort of users and non-users) [23]. As 38\% of the women recruited in the present study reported using oral contraception, we used the IOM's probability values and associated levels of usual intake for a mixed group of oral contraceptive users and non-users. For example, the probability that usual dietary iron intakes of 6.56 to $7.13 \mathrm{mg} /$ day are inadequate is 0.65 , whereas the probability that usual intakes ranging from 12.5 to $14.85 \mathrm{mg} /$ day are inadequate is 0.08 . The number of participants with usual iron intakes in each of the 14 levels was counted, converted to a percentage of the cohort, and then multiplied by the associated probability value for each level, generating the proportion of women with increased risk of inadequate intakes.

As zinc requirements are assumed to be normally distributed, participants' risk of inadequate zinc intakes was estimated using the EAR cut-point method [23]. The number of participants with usual dietary zinc intakes below the Australian EAR for zinc for women aged 19-50 years ( $<6.5 \mathrm{mg} /$ day [31]) was summed and presented as $n(\%)$.

\subsubsection{Biochemistry}

In this study, serum ferritin and hemoglobin were used as markers of iron status and serum zinc was used for zinc status. C-reactive protein (CRP) was used to indicate the presence of acute infection or 
inflammation. For women recruited in Melbourne, fasting venous blood samples were used for all biomarkers, and Dorevitch Pathology (Heidelberg, Melbourne, Australia) conducted analyses of serum ferritin, hemoglobin, and CRP. Serum ferritin was measured using the ADVIA Centaur Ferritin Assay on the Siemens ADVIA Centaur (Siemens Healthcare Diagnostics, Deerfield, Illinois, US), hemoglobin measured using the Sysmex Automated Hematology Analyzer XE-2100 (Sysmex, Kobe, Japan), and CRP measured using a latex-enhanced immunoturbidimetric assay on a Siemens ADVIA 2400 (Bayer Diagnostics, Tarrytown, NY, US). Women recruited at the Blood Service were nonfasting as data collection occurred just prior to making a blood donation, and venous samples were used for analyses except for hemoglobin, which used capillary samples. For women recruited at the Blood Service, the Blood Service conducted analyses of serum ferritin, hemoglobin, and CRP. Serum ferritin was measured using the AxSYM Ferritin assay on the AxSYM (Abbott Diagnostics, Abbott Park, IL, US), fingerprick hemoglobin was measured using the Hemocue B-Haemoglobin Photometer (Hemocue, Angelholm, Sweden), and CRP was measured using the Quantikine Human CRP Immunoassay (R \& D Systems, Minneapolis, MN, US).

For measurement of serum zinc concentration, a venous blood sample was collected in a trace element-free vacutainer (cat. no. 368380, BD Australia, North Ryde, Sydney, Australia). For women in Melbourne, sampling took place between 07:40 and 18:30; for women in Sydney, sampling took place between 08:00 and 16:55. Serum was separated and stored at $-70{ }^{\circ} \mathrm{C}$ in trace element-free microcentrifuge tubes (cat. no. 3013-870-000, Labcon, Ballarat, Australia) prior to transport and analysis for serum zinc in the Trace Element Laboratory at the Department of Human Nutrition, University of Otago, Dunedin, New Zealand. Serum zinc was measured using a ContrAA 700 contiuum source flame atomic absorption spectrometer (Analytik Jena AG, Jena, Germany) following a modified method of Smith et al. [32]. Serial replicates of an in-house pooled serum and quality control sera (UTAK, UTAK Laboratories, Valencia CA) were used to check the precision and accuracy of the assay. Samples were analyzed in two batches - those collected from 2010 to 2011 were analyzed in 2011, and those collected from 2012 to 2014 were analyzed in 2014. For the first batch, the interassay CV (as \%) for serum zinc was $3.5 \%(n=17)$, and the analyzed mean value for the zinc serum quality control was $10.4 \mu \mathrm{mol} / \mathrm{L}$ (CV 5.4\%, $n=12$ ) compared with the manufacturer's certified mean (SD) value of 10.4 (2.6) $\mu \mathrm{mol} / \mathrm{L}$. The interassay CV in the second batch was $2.2 \%(n=6)$, and the analyzed mean value for the quality control was $10.2 \mu \mathrm{mol} / \mathrm{L}(2.4 \%, n=3)$ compared with the manufacturer's certified mean (SD) value of 10.1 (2.4) $\mu \mathrm{mol} / \mathrm{L}$. Serum zinc concentrations were standardized to 08:00 blood sampling using the method of Arsenault et al. [33]. Briefly, serum zinc concentrations were standardized using a linear regression model with serum zinc concentration as the dependent variable and blood sampling time in hours as a covariate to obtain the $\beta$ coefficient for sampling time. Each participant's sampling time was centered around 08:00 and multiplied by the time coefficient, and this value was then subtracted from each woman's raw serum zinc concentration. These resulting standardized values were interpreted according to fasting status.

The reference values used to categorize biomarker data are presented in Table 1.

Complete iron and zinc biochemical data were available for 333 women. Seven of these women were excluded from descriptive and inferential analyses of iron and zinc status-one woman who had recently had an intravenous iron infusion (serum ferritin $461 \mu \mathrm{g} / \mathrm{L}$ ) and six women who had serum zinc concentrations exceeding $18.97 \mu \mathrm{mol} / \mathrm{L}$, indicative of potential contamination [34]. 
Table 1. Reference values used to interpret biomarkers of iron and zinc status.

\begin{tabular}{|c|c|c|c|c|}
\hline \multirow{2}{*}{ Classification } & \multicolumn{4}{|c|}{ Biomarker } \\
\hline & Serum Ferritin & Hemoglobin & CRP & Serum Zinc \\
\hline Depleted iron stores ${ }^{a}$ & $<15 \mu \mathrm{g} / \mathrm{L}$ & $\geq 120 \mathrm{~g} / \mathrm{L}$ & $<5 \mathrm{mg} / \mathrm{L}$ & - \\
\hline Iron overload ${ }^{\mathrm{b}}$ & $>150 \mu \mathrm{g} / \mathrm{L}$ & - & $<5 \mathrm{mg} / \mathrm{L}$ & - \\
\hline Anemia $^{a}$ & - & $<120 \mathrm{~g} / \mathrm{L}$ & - & - \\
\hline Iron-deficiency anemia $^{\text {a }}$ & $<15 \mu \mathrm{g} / \mathrm{L}$ & $<120 \mathrm{~g} / \mathrm{L}$ & $<5 \mathrm{mg} / \mathrm{L}$ & - \\
\hline Non-iron deficiency anemia & $\geq 15 \mu \mathrm{g} / \mathrm{L}$ & $<120 \mathrm{~g} / \mathrm{L}$ & $<5 \mathrm{mg} / \mathrm{L}$ & \\
\hline Low serum zinc ${ }^{c}$ & - & - & - & $\begin{array}{c}\text { AM fasting: }<10.7 \mu \mathrm{mol} / \mathrm{L} \\
\text { AM nonfasting: }<10.1 \mu \mathrm{mol} / \mathrm{L}\end{array}$ \\
\hline Inflammation/ infection ${ }^{\mathrm{d}}$ & - & - & $\geq 5 \mathrm{mg} / \mathrm{L}$ & - \\
\hline
\end{tabular}

\subsection{Statistical Analysis}

All statistical analyses were completed using Stata/SE 12.0 (StataCorp, TX, US), and for all tests, the significance level was set at $p<0.05$.

The normality of continuous variables was assessed by evaluating histograms, with normally distributed data presented as mean (SD) and skewed data presented as median (interquartile range (IQR; 27th to 75th centiles) and geometric mean (95\% CI)). Categorical data are presented as $n$ (\%). To compare groups, independent samples t-tests were used for normally distributed data, Wilcoxon rank-sum tests were used for skewed data, Pearson chi-square tests were as used for categorical data with expected counts $\geq 5$, and Fishers exact tests were used for categorical data with expected counts $<5$.

Nested linear regression analyses were used to investigate associations between dietary iron intake (mg/day; dependent variable) and dietary zinc intake (mg/day; independent variable), with and without energy intake (MJ/day) as a potential confounding variable. Inspection of residual-versus-fitted and component-plus-residual plots was conducted to confirm adherence to linear regression assumptions, and the fit of the nested models was compared using a likelihood ratio test. DfBeta statistics were produced and the regression analysis was repeated omitting any participants whose DfBeta statistic indicated disproportionate influence on the dietary zinc intake regression coefficient (cut-off DfBeta $>2 / \sqrt{ }$ n [37]).

To investigate associations between inadequate dietary iron and zinc intakes, women were dichotomized to those meeting the zinc EAR and those not meeting the zinc EAR, and the percentage of participants at risk of inadequate iron intakes in each group was compared using a two-proportion z-test (an immediate two-sample proportion test).

Nested linear regression analyses were also used to investigate continuous associations between uncorrected serum ferritin $(\mu \mathrm{g} / \mathrm{L})$ as the dependent variable and serum zinc ( $\mu \mathrm{mol} / \mathrm{L}$, standardized to 08:00 blood sampling) as the independent variable, with and without potential confounding variables (fasting status, CRP (mg/L), age, BMI, use of oral contraception, mL of blood donated over the past 12 months, use of dietary supplements containing iron and/or zinc). Plotting the residuals against the fitted values showed heteroscedasticity, therefore serum ferritin was natural log transformed for these 
analyses. The fit of these nested models were also compared using the likelihood ratio test and analysis was repeated omitting women whose DfBeta value indicated undue influence on the serum zinc regression coefficient. Logistic regression was used to investigate whether having depleted iron stores was associated with low serum zinc concentrations, adjusting for the potential confounding variables.

\section{Results}

\subsection{Characteristics of Participants}

Characteristics of study participants are shown in Table 2. Of the 382 women included in the study, 320 women had both dietary data and biochemical data, 59 women had only dietary data, and 3 women had only biochemical data. Eighty-one percent of women were tertiary educated, and $43 \%$ had made a blood donation in the previous year. Two-thirds of the cohort had BMI values in the normal range ( $n=258)$, and $28 \%(n=107)$ were overweight or obese.

Table 2. Demographic and anthropometric characteristics of 382 women who participated in the study.

\begin{tabular}{|c|c|}
\hline & $N=382$ \\
\hline \multicolumn{2}{|l|}{ Demographics } \\
\hline Age, years ${ }^{a}$ & $28.7(7.3)$ \\
\hline Tertiary education, $n$ (\%) & $307(81 \%)$ \\
\hline Current smoker, $n(\%)$ & $27(7 \%)$ \\
\hline Donated blood in previous 12 months, $n$ (\%) & $162(43 \%)$ \\
\hline Oral contraceptive use, $n$ (\%) & $146(38 \%)$ \\
\hline On 'special' diet, $n$ (\%) & 87 (23\%) \\
\hline Vegetarian, $n(\%)$ & $16(4.2 \%)$ \\
\hline Mostly vegetarian but eat some meats, $n$ (\%) & $33(9 \%)$ \\
\hline Other diets, $n(\%)^{\mathrm{b}}$ & $38(10 \%)$ \\
\hline \multicolumn{2}{|l|}{ Anthropometrics } \\
\hline Weight, kg a & $66.1(11.7)$ \\
\hline Height, $\mathrm{m}^{\text {a }}$ & $1.66(0.06)$ \\
\hline Body mass index, $\mathrm{kg} / \mathrm{m}^{2}$ a & $23.9(3.9)$ \\
\hline
\end{tabular}

\subsection{Intakes of Iron and Zinc}

Participants' usual intakes of dietary and supplemental iron and zinc are described in Table 3. Average usual dietary iron and zinc intakes were $10.5 \mathrm{mg} /$ day (range 1.8 to $25 \mathrm{mg} /$ day) and $9.3 \mathrm{mg} /$ day (range 1.6 to $40.9 \mathrm{mg} /$ day) respectively. Thirty-one percent of this cohort were at risk of inadequate dietary iron intakes, and 19\% were at risk of inadequate dietary zinc intakes. Twenty percent (73/373 women) were taking supplemental iron as either iron-specific or multivitamin/mineral supplements, and three women were taking both iron-specific and multivitamin/mineral supplements. Fourteen percent (52/370) were taking some form of supplemental zinc. Only 2/8 participants taking zinc-specific supplements provided 
dose information and these supplements contributed 25 to $50 \mathrm{mg} /$ day of elemental zinc. The dose of zinc from multivitamin/mineral supplements ranged from 0.4 to $30 \mathrm{mg} / \mathrm{day}$.

Table 3. Dietary and supplemental iron and zinc intakes in women aged 18-50 years $(N=379)$.

\begin{tabular}{|c|c|c|c|}
\hline & $\begin{array}{c}\text { Mean (SD) or } \\
n(\%) \\
\end{array}$ & Median (IQR) & $\begin{array}{c}\text { Geometric } \\
\text { Mean }(95 \% \mathrm{CI}) \\
\end{array}$ \\
\hline Dietary iron intake, mg/day & $10.5(3.5)$ & & \\
\hline At risk of inadequate dietary iron intake, $n(\%)^{\text {a }}$ & $117(31 \%)$ & & \\
\hline Iron-specific supplements, $n(\%)$ & $32(8 \%)$ & & \\
\hline Elemental iron, mg/day ${ }^{\mathrm{b}}$ & & 13.3 (5 to 30$)$ & 12.1 (6.9 to 21.2$)$ \\
\hline Vitamin/mineral supplements with iron, $n$ (\%) & $44(12 \%)$ & & \\
\hline Elemental iron, mg/day ${ }^{c}$ & & 5 (4.9 to 5$)$ & 4.6 (3.4 to 6.2$)$ \\
\hline Dietary zinc intake, mg/day & $9.3(3.8)$ & & \\
\hline At risk of inadequate dietary zinc intake, $n(\%)^{d}$ & $72(19 \%)$ & & \\
\hline Zinc-specific supplements, $n(\%)^{\mathrm{e}}$ & $8(2 \%)$ & & \\
\hline Vitamin/mineral supplements with zinc, $n$ (\%) & $45(12 \%)$ & & \\
\hline Elemental zinc (mg/day) ${ }^{\mathrm{f}}$ & & $6.3(4$ to 10$)$ & $5.4(4.1$ to 7.2$)$ \\
\hline
\end{tabular}

$\mathrm{IQR}=$ interquartile range (25th to 75 th centiles). ${ }^{\text {a }}$ Assessed using probability approach. ${ }^{\mathrm{b}}$ Supplemental iron dose data unavailable for 5/32 women taking iron-specific supplements. ${ }^{c}$ Supplemental iron dose data unavailable for $1 / 44$ women taking multivitamin/mineral supplements. ${ }^{\mathrm{d}}$ Assessed by cut-point method using Australian Estimated Average Requirement for women 19-50 years (6.5 mg/day [31]). ${ }^{\text {e }}$ Supplement zinc dose data only available for $2 / 8$ women taking zinc-specific supplements $\left(25 \mathrm{mg} /\right.$ day and $50 \mathrm{mg} /$ day). ${ }^{\mathrm{f}}$ Supplement zinc dose data unavailable for $1 / 45$ women taking multivitamin/mineral supplements.

Associations between Usual Dietary Iron and Zinc Intakes

There was a positive association between dietary iron intake and dietary zinc intake. Multiple linear regression indicated that for every $1 \mathrm{mg}$ /day increase of dietary iron intake, mean dietary zinc intake increased by $0.7 \mathrm{mg} /$ day, and $65 \%$ of variation in dietary iron and zinc intakes could be accounted for by each other (Table 4). After adjusting for energy intake, each $1 \mathrm{mg} /$ day increase of dietary iron intake was associated with a $0.4 \mathrm{mg} /$ day increase in dietary zinc intake (Table 4). A likelihood ratio test indicated that adjusting for energy intake improved the fit of the model (likelihood ratio $\chi^{2}=60.18$, $p<0.001$ ). Omitting nine women identified using DfBetas as being overly influential observations from the adjusted model did not markedly change results, as a $1 \mathrm{mg} /$ day increase in dietary iron intake still increased mean dietary zinc intake by $0.4 \mathrm{mg} /$ day (95\% CI 0.3 to 0.5 ).

Table 4. Multiple regression analysis of associations between dietary iron and zinc intakes in women aged $18-50$ years $(N=379)^{\text {a }}$.

\begin{tabular}{ccc}
\hline Model & $\boldsymbol{\beta ( 9 5 \% ~ C I ) ~})^{\mathbf{b}}$ & $\boldsymbol{p}$-Value \\
\hline $\begin{array}{c}\text { Unadjusted model } \\
\text { Adj. } R^{2}=0.648, p<0.001\end{array}$ & $0.739(0.684$ to 0.794$)$ & $<0.001$ \\
\hline $\begin{array}{c}\text { Adjusted model } \\
\text { c }\end{array}$ & $0.433(0.359$ to 0.507$)$ & $<0.001$ \\
Adj. $R^{2}=0.730, p<0.001$ & & \\
\hline
\end{tabular}

a In all models, dietary iron intake (mg/day) was the dependent variable and dietary zinc intake (mg/day) was the independent variable. ${ }^{\mathrm{b}}$ Unstandardized $\beta$-coefficient. ${ }^{\mathrm{c}}$ Adjusted for energy intake (MJ/day). 
Using a two-proportion z-test, the proportion of women at risk of inadequate dietary iron intakes was 63\% (95\% CI 52 to 74\%) among those with dietary zinc intakes below the EAR versus 23\% (95\% CI 18 to $28 \%)$ among those meeting the zinc $\operatorname{EAR}(p<0.001)$

\subsection{Biochemical Iron and Zinc status}

Details of participants' iron and zinc biomarkers are provided in Table 5. For the 32 women with acute inflammation, serum ferritin was multiplied by a factor of 0.65 [38]. After this correction for inflammation, thirty-seven percent of women had depleted iron stores, of which $18 \%$ had iron-deficiency anemia ( $7 \%$ of the total sample). Seventeen percent of the sample (57/326) had low serum zinc concentrations. Chi-square analysis indicated twenty-five percent (31/122) of women using oral contraception had low serum zinc concentrations compared to $13 \%$ of nonusers $(26 / 203) \chi^{2}(1, N=326)=8.37, p=0.004$. Women with fasting samples and women with nonfasting samples did not differ in terms of oral contraceptive use (38\% of fasting group using oral contraception vs. 37\% of nonfasting group) $\chi^{2}(1, N=325)=0.006, p=0.941$.

Table 5. Biochemical measures of iron and zinc status in women aged 18-50 years $(N=326)$.

\begin{tabular}{|c|c|c|c|}
\hline & $n(\%)$ & $\begin{array}{c}\text { Median (IQR) or } \\
\text { Mean (SD) } \\
\end{array}$ & $\begin{array}{c}\text { Geometric Mean } \\
(95 \% \mathrm{CI}) \\
\end{array}$ \\
\hline Serum ferritin, $\mu \mathrm{g} / \mathrm{L}^{\mathrm{a}}$ & & 21 (11 to 38$)$ & $19.0(17.2$ to 21.1$)$ \\
\hline $\mathrm{CRP}, \mathrm{mg} / \mathrm{L}$ & & $0.63(0.17$ to 2.11$)$ & $0.65(0.55$ to 0.77$)$ \\
\hline Hemoglobin, g/L $(n=143)$ & & $132(10)$ & \\
\hline Depleted iron stores, $n(\%)$ & $97(30 \%)$ & & \\
\hline Iron-deficiency anemia, $n(\%)^{\mathrm{a}, \mathrm{c}}$ & $22(7 \%)$ & & \\
\hline Non-iron deficiency anemia, $n(\%)^{d}$ & $7(2 \%)$ & & \\
\hline Iron overload, $n(\%){ }^{\mathrm{e}}$ & $2(0.6 \%)$ & & \\
\hline Elevated CRP, $n(\%){ }^{\mathrm{f}}$ & $32(10 \%)$ & & \\
\hline Fasting serum zinc, $\mu \mathrm{mol} / \mathrm{L}(n=143)^{\mathrm{g}}$ & & $12.6(1.7)$ & \\
\hline Low fasting serum zinc, $n(\%)^{\mathrm{h}}$ & $17(12 \%)$ & & \\
\hline Nonfasting serum zinc, $\mu \mathrm{mol} / \mathrm{L}(n=183)^{\mathrm{g}}$ & & $11.8(2.0)$ & \\
\hline Low nonfasting serum zinc, $n(\%)^{\mathrm{h}}$ & $40(22 \%)$ & & \\
\hline \multicolumn{4}{|c|}{ 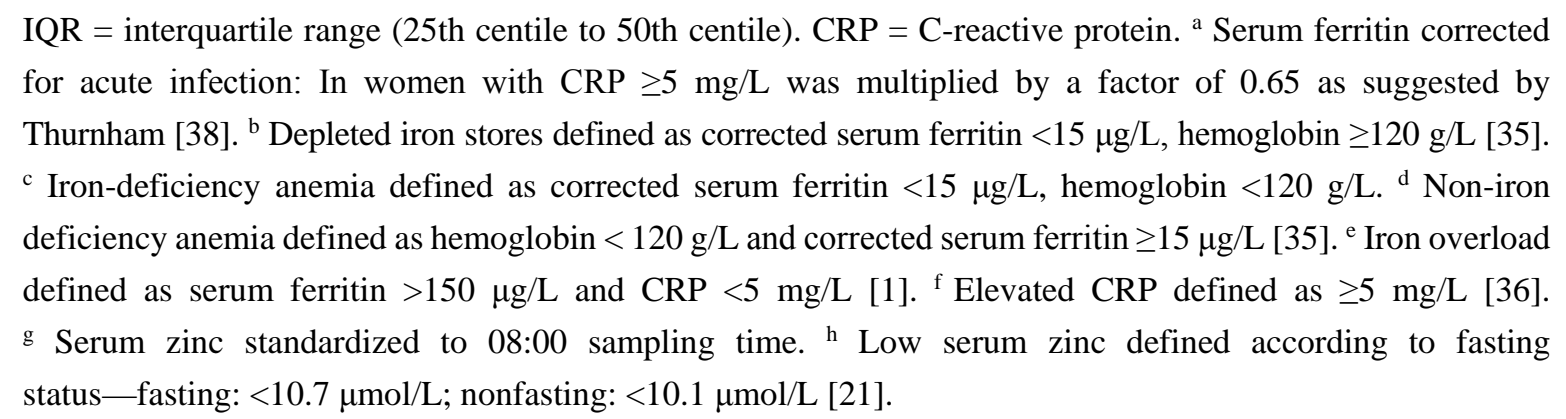 } \\
\hline
\end{tabular}

Associations between Iron and Zinc Status

There was a small positive association between serum ferritin and serum zinc concentrations. Simple linear regression of natural log transformed serum ferritin (not corrected for acute infection) on serum zinc indicated that an increase of $1 \mu \mathrm{mol} / \mathrm{L}$ in serum zinc was associated with a $10 \%$ increase in mean 
serum ferritin (Table 6). This association decreased slightly after adjusting for fasting status, CRP, age, BMI, use of oral contraception, blood donation, and use of iron and/or zinc supplements, so that an increase of $1 \mu \mathrm{mol} / \mathrm{L}$ in serum zinc was associated with a $6.4 \%$ increase in mean serum ferritin (Table 6). A greater proportion of the variance was explained by the adjusted model compared to the unadjusted model, and a likelihood ratio test indicated that the fit of the adjusted model was better than that of the unadjusted model (likelihood ratio $\chi^{2}=60.47, p<0.001$ ).

A more conservative model omitting 16 women identified as having disproportionate influence on the serum zinc regression coefficient did not change the findings, with $1 \mu \mathrm{mol} / \mathrm{L}$ in serum zinc associated with a $5.7 \%$ increase in mean serum ferritin (antilogarithm $\beta$ 95\% CI 1.004 to 1.11 ).

Table 6. Multiple regression analysis of associations between serum ferritin and serum zinc concentrations in women aged $18-50$ years $(N=326)^{\text {a }}$.

\begin{tabular}{|c|c|c|c|}
\hline & $\begin{array}{c}\text { Logarithmic Scale } \beta \\
(95 \% \text { CI })^{\text {b }}\end{array}$ & $\begin{array}{c}\text { Antilogarithm of } \beta \\
(95 \% \mathrm{CI})\end{array}$ & $p$-Value \\
\hline $\begin{array}{c}\text { Unadjusted model } \\
\text { Adj. } R^{2}=0.035, p<0.001\end{array}$ & $0.096(0.043$ to 0.148$)$ & 1.100 (1.043 to 1.160$)$ & $<0.001$ \\
\hline $\begin{array}{c}\text { Adjusted model }^{\mathrm{c}} \\
\text { Adj. } R^{2}=0.181, p<0.001\end{array}$ & 0.061 ( 0.010 to 0.112$)$ & 1.063 (1.010 to 1.118 ) & 0.019 \\
\hline
\end{tabular}

No association was observed between low serum ferritin and low zinc levels in this cohort. Fifteen percent (32/213) of women with adequate iron stores presented with low serum zinc concentration, compared to $22 \%$ (25/113) of women with depleted iron stores, however these proportions were not statistically different $\chi^{2}(1, N=326)=2.58, p=0.108$. Furthermore, using logistic regression to account for fasting status, CRP, oral contraceptive use, and supplement use found women with low serum zinc concentrations were not at increased risk of depleted iron stores compared to women with normal serum zinc concentrations (odds ratio for having depleted iron stores if having low serum zinc $=1.37, p=0.340$ (95\% CI 0.72 to 2.64$)$ ).

\section{Discussion}

We found a significant positive relationship between usual dietary iron intakes and dietary zinc intakes in our cohort of Australian premenopausal women. Each $1 \mathrm{mg} /$ day increase in dietary iron intake corresponded with a $0.4 \mathrm{mg} /$ day increase in dietary zinc intake. Furthermore, women with dietary zinc intakes below Australian recommendations (EAR of $6.5 \mathrm{mg} /$ day [31]) were at greater risk of inadequate dietary iron intakes as determined using the probability approach (63\% at risk vs. $23 \%$ at risk). Our findings support observations in other cohorts that food sources of zinc are also important food sources of iron [6,7], and also supports the theory that usual low intakes of either mineral increase the likelihood of low intakes of the other [8]. 
We also observed a small positive association between biochemical iron and zinc status, with a $1 \mu \mathrm{mol} / \mathrm{L}$ increase in serum zinc corresponding to a six percent increase in serum ferritin. This finding is in line with previous research that also reported a positive association [14,16,17], however in our cohort, women with biomarkers below internationally-recognized cut-offs for low levels of one mineral were not more likely to present with low levels of the other mineral. Together, these findings suggest that while there may be a positive relationship between iron and zinc status at a population level, it may be too small to translate to a clinically meaningful association among women in Australia. Serum ferritin is not a useful proxy for serum zinc concentrations in our study setting, and women with depleted iron stores are not at increased risk of impaired zinc status. Given our findings that $17 \%$ of our cohort are at risk of zinc deficiency and given the health consequences associated with impaired zinc status $[19,20]$, further work is needed to determine the zinc status of women in the Australian population.

Our observation of a strong association between usual iron and zinc intakes was not accompanied by a similarly strong association between iron and zinc status, possibly reflecting the nutrients' different responses to potential inhibitors and enhancers of absorption. Prosser et al. investigated the effect of a four-month dietary iron intervention on serum zinc of premenopausal women [17]. Women with low iron stores were randomized to a group receiving $50 \mathrm{mg}$ /day supplemental iron, a placebo group, or a whole-diet intervention designed to increase iron bioavailability [39]. Women in the dietary intervention did not change their dietary iron or dietary zinc intakes, but their intakes of meat/fish/poultry, heme iron, and vitamin $C$ increased and phytate intakes decreased [17,39]. This diet improved serum ferritin by 26\% [39], however it had no effect on serum zinc concentrations compared to the placebo group [17]. An increase in serum ferritin concentration via dietary means is not necessarily partnered with an increase in serum zinc concentration, perhaps as factors influencing the proportion of iron available for absorption are not as influential on the bioavailability of zinc, and an increase in absolute zinc intake might also be needed to increase serum zinc in women. Although we found a strong association between dietary iron and zinc intakes, it seems targeted advice specific for each nutrient is required, and bioavailability of both cannot be improved through the same dietary means.

In contrast to previous research investigating both iron status and zinc status of women in Western countries [14-16], we incorporated an acute inflammatory marker in our analyses. Using a regression model that included CRP only slightly attenuated the association between iron and zinc status, perhaps due to the small proportion of women with elevated CRP in our sample (10\%). In other groups, measuring and correcting for CRP may be prudent if investigating a relationship between iron and zinc status. The distribution of CRP concentrations in the present sample falls below the distribution observed among women from the National Health and Nutrition Examination Survey (NHANES) 1999-2000 aged 20-49 years (median CRP approximately $2 \mathrm{mg} / \mathrm{L}$ (IQR 0.8 to $6 \mathrm{mg} / \mathrm{L}$ )) [40]. In this NHANES cohort, at least $25 \%$ of women had CRP $>5 \mathrm{mg} / \mathrm{L}$, and in such groups who are more prone to inflammation and infection, correcting for CRP may be more important. Where risk of inflammation is unknown, we suggest erring on the side of measuring CRP.

Our investigation supports findings from previous research suggesting a small statistical association between iron and zinc status in premenopausal women [14-17]. The strengths of this present study was use of internationally recognized biomarkers for iron status and zinc status, and use of conservative statistical analyses. Limitations include the sample of participants perhaps not being representative of premenopausal women in Australia (e.g., high proportion of women with tertiary education [41], 
majority with BMI values within the normal range [42], lower proportion of current smokers [42]) and use of a FFQ to assess adequacy of usual dietary iron and zinc intakes [43], although correlations for dietary iron intake in the previous version of our FFQ were comparable to that found in the validation of the Block FFQ against two four-day diet records ( $r=0.34$ to 0.42 [44]) and the validation of the Willett FFQ against a one-year diet record ( $r=0.47$ [43]) (validation of these two FFQs for dietary zinc intakes has not been published). We acknowledge that the IOM values used to conduct the probability method of assessing usual dietary iron intake may not represent the iron requirements of our cohort as there was a greater prevalence of oral contraceptive use among our participants than proposed by the IOM, and the IOM values do not account for blood donation. Our assessment of risk of inadequacy associated with dietary intakes must therefore be interpreted with caution. The use of oral contraception may also inflate our prevalence of low serum zinc concentrations, as oral contraceptives are known to suppress serum zinc concentration via hemodilution [21]. In addition, the present analysis of associations between iron and zinc status did not account for menstrual blood loss, a known influence in iron status [45]. To confirm the findings of our study, population-based studies assessing biochemical iron and zinc status and using rigorous dietary assessment methods are required, as is information on how current rates of oral contraceptive use and blood donation might affect women's iron and zinc requirements.

\section{Conclusions}

In conclusion, we found positive associations between usual dietary iron and zinc intakes in premenopausal women, with those meeting dietary zinc recommendations being less likely to have inadequate iron intakes compared to those not meeting zinc recommendations. We also found a small positive association between iron status and zinc status in the same cohort, although this association may not be clinically meaningful. Our data do not support the ability to identify women at risk of impaired zinc status through exploration of iron status, and further work understanding the zinc status of women within the Australian population is required.

\section{Acknowledgments}

Karen Lim is supported by a Deakin University Postgraduate Research Scholarship. This study was funded by Deakin University and the Australian Red Cross Blood Service, and supported by a competitive, peer-reviewed grant from Meat and Livestock Australia Ltd. Meat and Livestock Australia Ltd. had no role in the design and conduct of the study; collection, management, and interpretation of the data; or preparation of the manuscript. The authors acknowledge Australian governments that fund the Australian Red Cross Blood Service for the provision of blood, blood products and services to the Australian community, and thank Graham Giles of the Cancer Epidemiology Centre of The Cancer Council Victoria for permission to use the Dietary Questionnaire for Epidemiological Studies (Version 3.1).

\section{Author Contributions}

L.R., C.N., and D.I. planned and initiated the study; all authors carried out the study; R.S.G. and K.B.B. conducted serum zinc analyses; K.L. wrote the first draft; L.R., C.N., E.A.S.-G., A.B., R.S.G. 
and K.L. interpreted the results; all authors were involved in critical revision of the manuscript and approved the final manuscript for submission.

\section{Conflicts of Interest}

C.N. is a member of World Action on Salt and Health and the Australian division of World Action on Salt and Health and does not receive any financial support from these organizations. C.N. has received remuneration from Meat and Livestock Australia Ltd., Nestle Health Science, and Dairy Health Consortium. These payments are unrelated to the submitted work. The remaining authors declare no conflict of interest.

\section{References}

1. World Health Organization. Serum Ferritin Concentrations of the Assessment of Iron Status and Iron Deficiency in Populations, 2011. Available online: http://www.who.int/vmnis/indicators/ ferritin/en/ (accessed on 22 September 2014).

2. Australian Bureau of Statistics. Nutrient biomarkers by ranges/Nutrient biomarkers for women aged 16-44 years by ranges, 2013. Available online: http://www.abs.gov.au/AUSSTATS/abs@.nsf/ DetailsPage/4364.0.55.0062011-12?OpenDocument (accessed on 6 February 2015).

3. Pasricha, S.R.; Low, M.; Thompson, J.; Farrell, A.; de-Regil, L.M. Iron supplementation benefits physical performance in women of reproductive age: A systematic review and meta-analysis. J. Nutr. 2014, 144, 906-914.

4. Murray-Kolb, L.E.; Beard, J.L. Iron treatment normalizes cognitive functioning in young women. Am. J. Clin. Nutr. 2007, 85, 778-787.

5. Leonard, A.J.; Chalmers, K.A.; Collins, C.E.; Patterson, A.J. A study of the effects of latent iron deficiency on measures of cognition: A pilot randomised controlled trial of iron supplementation in young women. Nutrients 2014, 6, 2419-2435.

6. Cole, C.R.; Grant, F.K.; Swaby-Ellis, E.D.; Smith, J.L.; Jacques, A.; Northrop-Clewes, C.A.; Caldwell, K.L.; Pfeiffer, C.M.; Ziegler, T.R. Zinc and iron deficiency and their interrelations in low-income African American and Hispanic children in Atlanta. Am. J. Clin. Nutr. 2010, 91, 1027-1034.

7. Lee, D.H.; Folsom, A.R.; Jacobs, D.R., Jr. Iron, zinc, and alcohol consumption and mortality from cardiovascular diseases: The Iowa Women’s Health Study. Am. J. Clin. Nutr. 2005, 81, 787-791.

8. Gibson, R.S.; Heath, A.L.; Ferguson, E.L. Risk of suboptimal iron and zinc nutriture among adolescent girls in Australia and New Zealand: Causes, consequences, and solutions. Asia Pacific J. Clin. Nutr. 2002, 11 (Suppl. 3), S543-S552.

9. Hunt, J.R. Bioavailability of iron, zinc, and other trace minerals from vegetarian diets. Am. J. Clin. Nutr. 2003, 78, 633s-639s.

10. Lim, K.H.; Riddell, L.J.; Nowson, C.A.; Booth, A.O.; Szymlek-Gay, E.A. Iron and zinc nutrition in the economically-developed world: A review. Nutrients 2013, 5, 3184-3211.

11. Hallberg, L. Bioavailability of dietary iron in man. Annu. Rev. Nutr. 1981, 1, 123-147.

12. Gibson, R.S. The role of diet- and host-related factors in nutrient bioavailability and thus in nutrientbased dietary requirement estimates. Food Nutr. Bull. 2007, 28, S77-S100. 
13. Solomons, N.W.; Jacob, R.A.; Pineda, O.; Viteri, F. Studies on the bioavailability of zinc in man. II. Absorption of zinc from organic and inorganic sources. J. Lab. Clin Med. 1979, 94, 335-343.

14. Gibson, R.S.; Heath, A.L.; Prosser, N.; Parnell, W.; Donovan, U.; Green, T.; McLaughlin, K.E.; O’Connor, D.; Bettger, W.; Skeaff, C.M. Are young women with low iron stores at risk of zinc as well as iron deficiency? In Trace Elements in Man and Animals 10; Roussel, A.M., Favier, A.E., Anderson, R.A., Eds.; Kluwer Academic Publishers: New York, NY, US, 1999; pp. 323-328.

15. Yokoi, K.; Alcock, N.W.; Sandstead, H.H. Iron and zinc nutriture of premenopausal women: Associations of diet with serum ferritin and plasma zinc disappearance and of serum ferritin with plasma zinc and plasma zinc disappearance. J. Lab. Clin. Med. 1994, 124, 852-861.

16. Yokoi, K.; Sandstead, H.H.; Egger, N.G.; Alcock, N.W.; Sadagopa Ramanujam, V.M.; Dayal, H.H.; Penland, J.G. Association between zinc pool sizes and iron stores in premenopausal women without anaemia. Br. J. Nutr. 2007, 98, 1214-1223.

17. Prosser, N.R.; Heath, A.L.; Williams, S.M.; Gibson, R.S. Influence of an iron intervention on the zinc status of young adult New Zealand women with mild iron deficiency. Br. J. Nutr. 2010, 104, $742-750$.

18. King, J.C. Zinc: An essential but elusive nutrient. Am. J. Clin. Nutr. 2011, 94, 679s-684s.

19. Shankar, A.H.; Prasad, A.S. Zinc and immune function: The biological basis of altered resistance to infection. Am. J. Clin. Nutr. 1998, 68, 447s-463s.

20. Lai, J.; Moxey, A.; Nowak, G.; Vashum, K.; Bailey, K.; McEvoy, M. The efficacy of zinc supplementation in depression: Systematic review of randomised controlled trials. J. Affect. Disord. 2012, 136, e31-e39.

21. Brown, K.H.; Rivera, J.A.; Bhutta, Z.; Gibson, R.S.; King, J.C.; Lonnerdal, B.; Ruel, M.T.; Sandtrom, B.; Wasantwisut, E.; Hotz, C. International Zinc Nutrition Consultative Group (IZiNCG) technical document \#1. Assessment of the risk of zinc deficiency in populations and options for its control. Food Nutr. Bull. 2004, 25, S99-S203.

22. World Health Organization. Mean Body Mass Index (BMI), 2015. Available online: http://www.who.int/gho/ncd/risk_factors/bmi_text/en/ (accessed on 6 February 2015).

23. Institute of Medicine, Food and Nutrition Board. Dietary Reference Intakes for Vitamin A, Vitamin K, Arsenic, Boron, Chromium, Copper, Iodine, Iron, Manganese, Molybdenum, Nickel, Silicon, Vanadium, and Zinc; National Academies Press: Washington, DC, USA, 2001.

24. Cancer Council Victoria. Dietary Questionnaire for Epidemiological Studies version 2: User guide, 2014. Available online: http://www.cancervic.org.au/downloads/cec/FFQs/Jan_2015_Update/ DQES_guide_22jan15.pdf (accessed on 13 March 2015).

25. Hodge, A.; Patterson, A.J.; Brown, W.J.; Ireland, P.; Giles, G. The Anti Cancer Council of Victoria FFQ: Relative validity of nutrient intakes compared with weighed food records in young to middle-aged women in a study of iron supplementation. Aust. N. Z. J. Public Health 2000, 24, 576-583.

26. Food Standards. NUTTAB 2010, 2014. Available online: http://www.foodstandards.gov.au/ science/monitoringnutrients/nutrientables/pages/default.aspx (accessed on 6 February 2015).

27. Food Standards. AUSNUT 2007, 2013. Available online: http://www.foodstandards.gov.au/ science/monitoringnutrients/ausnut/Pages/ausnut2007.aspx (accessed on 6 February 2015). 
28. Lioret, S.; McNaughton, S.A.; Spence, A.C.; Crawford, D.; Campbell, K.J. Tracking of dietary intakes in early childhood: The Melbourne InFANT Program. Eur. J. Clin. Nutr. 2013, 67, 275-281.

29. Spence, A.C.; McNaughton, S.A.; Lioret, S.; Hesketh, K.D.; Crawford, D.A.; Campbell, K.J. A health promotion intervention can affect diet quality in early childhood. J. Nutr. 2013, 143, 1672-1678.

30. National Research Council. Nutrient Adequacy: Assessment Using Food Consumption Surveys; The National Academies Press: Washington, DC, USA, 1986.

31. National Health and Medical Research Council. Nutrient Reference Values for Australia and New Zealand Including Recommended Dietary Intakes; National Health and Medical Research Council: Canberra, Australia, 2006.

32. Smith, J.C., Jr.; Butrimovitz, G.P.; Purdy, W.C. Direct measurement of zinc in plasma by atomic absorption spectroscopy. Clin. Chem. 1979, 25, 1487-1491.

33. Arsenault, J.E.; Wuehler, S.E.; de Romana, D.L.; Penny, M.E.; Sempertegui, F.; Brown, K.H. The time of day and the interval since previous meal are associated with plasma zinc concentrations and affect estimated risk of zinc deficiency in young children in Peru and Ecuador. Eur. J. Clin. Nutr. 2011, 65, 184-190.

34. Engle-Stone, R.; Ndjebayi, A.O.; Nankap, M.; Killilea, D.W.; Brown, K.H. Stunting prevalence, plasma zinc concentrations, and dietary zinc intakes in a nationally representative sample suggest a high risk of zinc deficiency among women and young children in Cameroon. J. Nutr. 2014, 144, 382-391.

35. World Health Organization; Centers for Disease Control and Prevention. Assessing the Iron Status of Populations, 2007. Available online: http://www.who.int/nutrition/publications/micronutrients/ anaemia_iron_deficiency/9789241596107/en/(accessed on 21 January 2015).

36. Thurnham, D.I.; McCabe, G.P. Influence of infection and inflammation on biomarkers of nutritional status with an emphasis on vitamin A and iron. In Report: Priorities in the Assessment of Vitamin A and Iron Status in Populations, Panama City, Panama, 15-17 September 2010; World Health Organization, Ed.; World Health Organization: Geneva, Switzerland, 2012.

37. Belsley, D.; Kuh, E.; Welsch, R. Regression Diagnostics: Identifying Influential Data and Sources of Collinearity; Wiley: New York, NY, USA, 1980.

38. Thurnham, D.I.; McCabe, L.D.; Haldar, S.; Wieringa, F.T.; Northrop-Clewes, C.A.; McCabe, G.P. Adjusting plasma ferritin concentrations to remove the effects of subclinical inflammation in the assessment of iron deficiency: A meta-analysis. Am. J. Clin. Nutr. 2010, 92, 546-555.

39. Heath, A.L.; Skeaff, C.M.; O’Brien, S.M.; Williams, S.M.; Gibson, R.S. Can dietary treatment of non-anemic iron deficiency improve iron status? J. Am. Coll. Nutr. 2001, 20, 477-484.

40. Ford, E.S.; Giles, W.H.; Mokdad, A.H.; Myers, G.L. Distribution and correlates of C-reactive protein concentrations among adult US women. Clin. Chem. 2004, 50, 574-581.

41. Australian Bureau of Statistics. Non-School Qualification at Bachelor Degree Level or above, Persons Aged 20-64 Years, 2014. Available online: http://www.abs.gov.au/AUSSTATS/abs@.nsf/ DetailsPage/6227.0May\%202014?OpenDocument (accessed on 11 February 2015). 
42. Australian Bureau of Statistics. Australian Health Survey: First Results, 2011-2012, 2012. Available online: http://www.abs.gov.au/AUSSTATS/abs@.nsf/DetailsPage/4364.0.55.001201112?OpenDocument (accessed on 11 February 2015).

43. Willett, W. Nutritional Epidemiology, 3rd ed.; Oxford University Press: New York, NY, US, 2013.

44. Block, G.; Woods, M.; Potosky, A.; Clifford, C. Validation of a self-administered diet history questionnaire using multiple diet records. J. Clin. Epidemiol. 1990, 43, 1327-1335.

45. Hallberg, L.; Hulthen, L.; Bengtsson, C.; Lapidus, L.; Lindstedt, G. Iron balance in menstruating women. Eur. J. Clin. Nutr. 1995, 49, 200-207.

(C) 2015 by the authors; licensee MDPI, Basel, Switzerland. This article is an open access article distributed under the terms and conditions of the Creative Commons Attribution license (http://creativecommons.org/licenses/by/4.0/). 